\title{
The possibility of kinetic typography expression in the Internet art museum
}

\author{
Yasufumi Uekita* \& Yasushi Harada** \& Masahiko Furukata*** \\ ${ }^{*}$ Institute of Art \& Design, University of Tsukuba \\ Tsukuba City, Ibaraki Pref., 305-0006, Japan \\ E-mail uekita@geijutsu.tsukuba.ac.jp \\ **Institute of Art \& Design, University of Tsukuba \\ Tsukuba City, Ibaraki Pref., 305-0006, Japan \\ E-mail yhard@geijutsu.tsukuba.ac.jp \\ *** International Media Research Foundation \\ Shibuya-ku, Tokyo,150, Japan \\ E-mail furu@imrf.or.jp
}

\section{ABSTRACT}

This research is aimed at investigating the method of use of kinetic typography in order to appreciate a work in the art museum built by the Internet. Kinetic typography is the animation of the character expressed by computer. With being accompanied by various motions, kinetic typography can add messages, such as feeling and directions, to the meaning which language involves.

The Internet art museum is expressed by virtual space, which has two or three dimentions, expressed by computer. The exhibit works in the Internet art museum could be linked to various information, without being restricted to space or time.

Kinetic typography may help to grasp the relation of ambiguous information clearly in such case. For example when the information related among some works, must be expresed, the kinetic typography with character motion attached to the work reminds the meaning of information associably.

Through the experiment, the attributes of kinetic typography had been indicated as ornamentation of motion, settlement of motion, motion classification, and timebase appearance. A motion is expression of some meanings and includes additional information. When a motion of kinetic typography is applied to the intention of a message, the motion can express a meaning effectively.

There is a method of making a motion of kinetic typography generates automatically with a script. This is a possibility of appreciating the Internet art museum which used kinetic typography on the network. "School of Athens" painted by Sanzio Raffaello was made into the sample in this research. Some kinetic typography scripted by $\mathrm{C}$-language are operated on this picture to show the information construction of the picture.

In some casestudies, kinetic typography was pointed out to be an effective tool in order to offer the information related to the works in the Internet art museum.

\section{THE PURPOSE OF RESEARCH}

In recent years, an increasing amount of attention has been paid to the device of kinetic typography for visual representation on a computer screen. It is pointed out that the motion of kinetic typography shows kinds of value such as feeling and directions.

Kinetic typography is often used for the web of the Internet with a visual effect. The exhibit work introduced by the Internet is usually explained by a figure and a sentence. The visual effect of kinetic typography may explain effectively the exhibit work in the Internet.
This research considers a possibility that kinetic typography will carry out effective expression in the Internet art museum. A motion of kinetic typography must be designed in order to visualize the structure of a meaning which the work has. The structure of the motion operated by computer is visualizing the contents of un-visual information.

Furthermore, the computer program of kinetic typography mmotion has a possibility of extending the character expression in the Internet.

\section{FEATURE OF KINETIC TYPOGRAPHY}

Kinetic typography is the animation of the character made by computer. Moving a character by computer can express feeling, expression of an image, and explanation of a situation.

The character written on paper can express information logically. A picture and animation can express an image directly rather than it expresses information logically. Therefore, kinetic typography may have a nature of both character and animation.

A computer monitor is an environment where character and animation are made to live together. When explaining a figure on the computer monitor, a figure and a sentence are written together as being written to books. In this case, an observer reads a sentcncc, undcrstands cxplanation, looks at a figure, applics with explanation, and grasps the whole meaning.

That kinetic typography points to a figure directly makes it a part of explanation of a figure recognized comprehensively. At this time, a motion of kinetic typography must express the composition of explaining a figure. That is, to design of kinetic typography needs to reflect the informational composition of explaining.

\section{FUNDAMENTAL STRUCTURE OF KINETIC MOTION}

Motions of kinetic typography have many patterns. The pattern of kinetic motion has 'movement', 'rotation', 'appearance', 'modification', of a simple substance character. Moreover, a pattern may express the relation of two or more characters. In this case, the resize of a character and the change of a motion make the visual effect relatively.

Since kinetic typography expressed the image visually, the sensuous word is expressed by it. Some Japanese sensuous words were selected as onomatopoeia and mimetic word. Fig. 1 shows a sample of the onomatopoeia of "giggle." For the experi- 

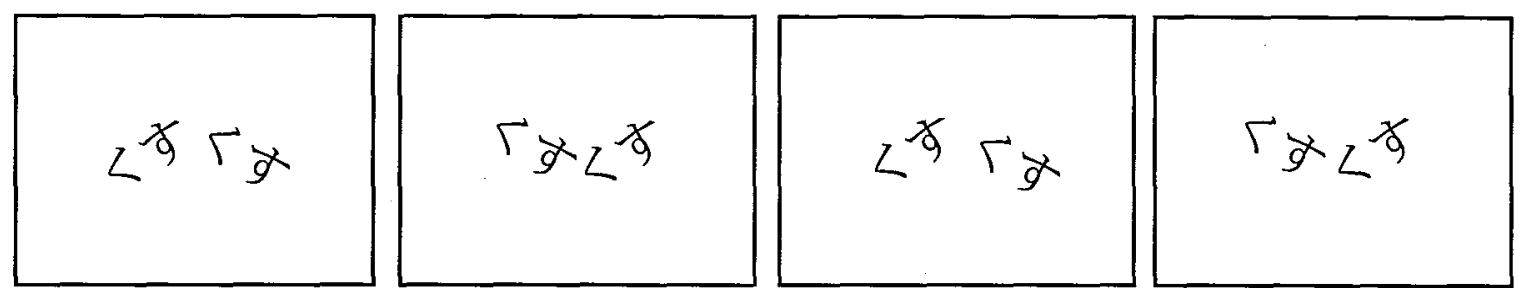

Fig.1 Sample of Japanese Kinetic Typography "giggle"

圆 never probably $\mathbf{D a}$ little $\mathbf{0}$ mostly $\mathbf{E}$ clear

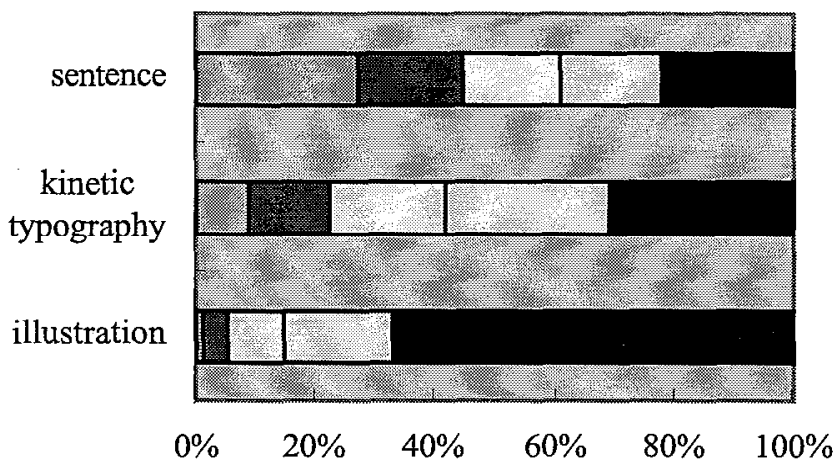

Fig.2 Ratio of Understanding by International Students

ment of kinetic typography, fourteen samples of sensuous word were made as a language-study application for hearing impairment children. The hearing impairment children can seldom be heard. Therefore, to study of onomatopoeia mimetic word is difficult for them. Kinetic typography may help them for understanding of those words. But this experiment will be tested later, so the result has not yet come.

As a prior research before the experipent for them, the image questionnaire of kinetic typography using this sample had been performed to the nineteen international students and the eighteen Japanese students about 20 years old. Some of international students did not know Japanese mimetic words and onomatopoeia. They looked at the kinetic typography and were going to understand the meanig of Japanese mimetic words and onomatopoeia. Since the Japanese students had known the meaning of Japanese mimetic word and onomatopoeia, they could judge the kinetic typography in the position in which a person did not understand the meanings, and would learn the onomatopoeia and mimetic words.

In the experiment, the observers looked at the sentences first, and watched the kinetic typography, and finally looked at the illustrations. Fig. 2 and 3 are the diagrams showing the impression effect of the kinetic typography. Through the both figures, the kinetic typography helps to understand the meaning of Japanese mimetic words and onomatopoeias. The illustrations contribute to understanding completely. As the result of this experiment, the kinetic typography could have the role of both logical sentence and image-illustration.

The motion classification of the kinetic typography is shown in the Fig. 4, 5,6. And the following thing is pointed out through this analysis of the motions.

1) A motion of kinetic typography becomes the settlement "character", "word" and "phrase," respectively. And sound-intonation may have influenced the settlement.

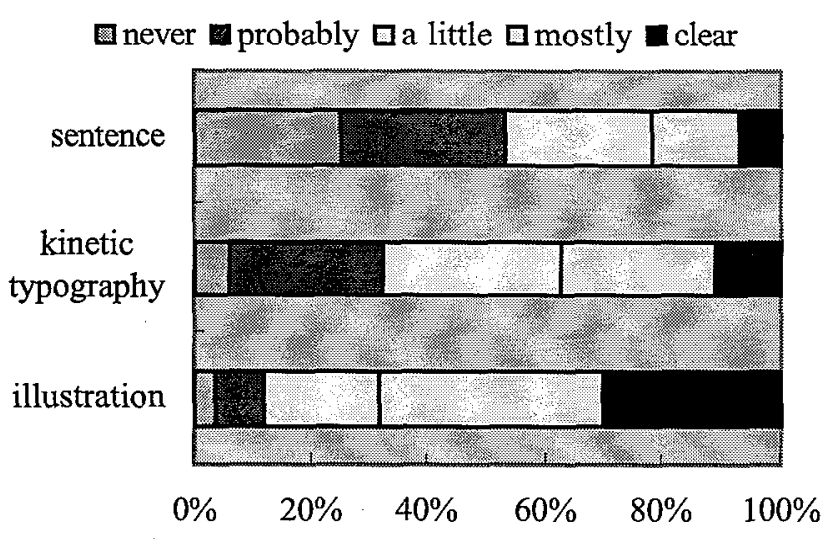

Fig.3 Ratio of Understanding by Japanese Students

2) A motion of kinetic typography can be divided into "linear motion" and "curvilinear motion" and "rotation." Linear motion is "upper and lower sides", "right and left." Curvilinear motion is "curvature" and "drifting irregularity." Rotation has a rotational centaral point.

3) Change of a character size is devided into "expansion" and "reduction."

4) Display way is devided into "continuous", "generation", "disappearance", and "blink."

5) A motion of kinetic typography has combined the elements shown above.

\section{DISPLAY FEATURE OF INTERNET ART MUSEUM}

In this research, the Internet art museum is assumed in the virtual art museum displayed on a computer screen. An advantage of the Internet art museum is able to appreciate many exhbit works in front of the screen, even if an observer does not go to an actual art museum directly. The work displayed on the screen of the Internet art museum is an image, and differs from the actual art work. When people appreciate in the Internet art museum, they do not necessarily demand the impression when seeing a real work in the art museum. It will be important for them to know the explanation of the exhibit work and to deepen understanding relating with it.

In order to know the exhibit work, generally people learn through a document. The document about the work carries the photograph and the explanatory note of it. Many present Internet art museums are explained by the same display method as same as a document written in book. When people read an explanatory note in the Internet art museum, they meet the document, and they 


$$
\begin{aligned}
& \text { character } \\
& \text { word } \\
& \text { phrase } \\
& \text { (sentence) } \\
& \underline{\mathrm{g}} \underline{\mathrm{i}} \underline{\mathrm{g}}_{\underline{1}} \underline{\mathrm{e}} \quad \underline{\mathrm{gi}} \underline{\mathrm{gle}}
\end{aligned}
$$

Fig.4 Motion Settlement of Kinetic Typography
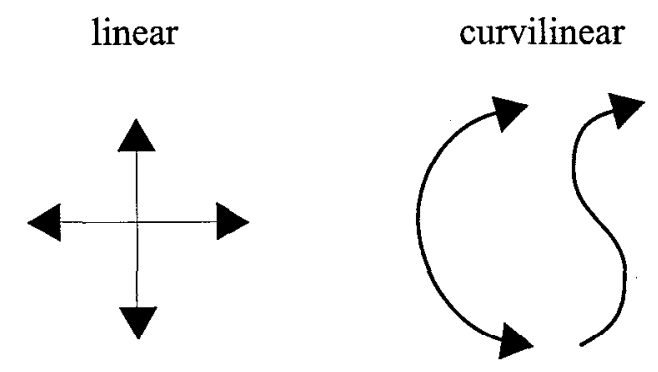

rotation

expansion

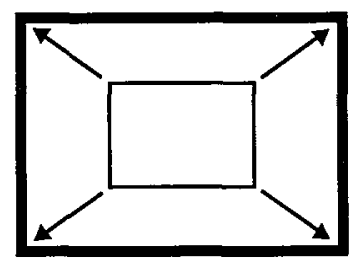

reduction

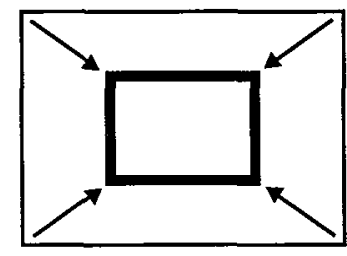

Fig.5 Motion Classification of Kinetic Typography

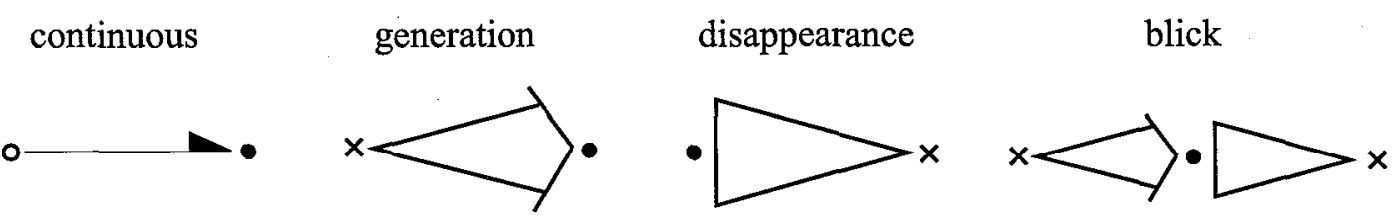

Fig.6 Timebase Appearance of Kinetic Typography

have to read the explanatory note.

They look at the work first., and they read the explanatory note and understand the logic of explanation of the work. Next, they look at the work again, and apply the contents of understanding to it.

Application of an interaction displaycd by computer may spread the expressional possibility more. The interaction by the computer provides explanation of exhibit works with the new appreciation method. The kinetic typography with the possibility of an interaction display can demonstrate in the Internet art museum.

As the method of interaction in the Internet art museum, "Related Exhibition" will be an effective form of many exhibition methods[2]. It is the method which shows the meaning of a work using the correlation of data. "Related exhibition" is the method of understanding the work through the reaction between data, a dialog and time transition, causal relationship and changes of a style, its influence, and action rebounding between works.

\section{INTERNET ART MUSEUM ACCOMPANIED WITH KINETIC TYPOGRAPHY}

"School of Athens" painted by Sanzio Raffaello was made into the sample in this research. This picture is the mural painting painted on the royal palace of Vatican at the beginning of the 16 th century. This motif and theme were the material interpreted logically, and will be suitable to explanation of related exhibition. And it cannot be carried out from the royal palace of the Vatican as a tableau. If we do not go there, we cannot appreciate the work.

The first feature of this picture is interpreted by the wholc and the portion. Masunari [3] cxplains as follows about the composition of the whole picture.

<The board written to be "recognition of a cause" and the lady 
Fig.7 Sample of Kinetic Typography showing the Whole Theme of the "School of Athens"
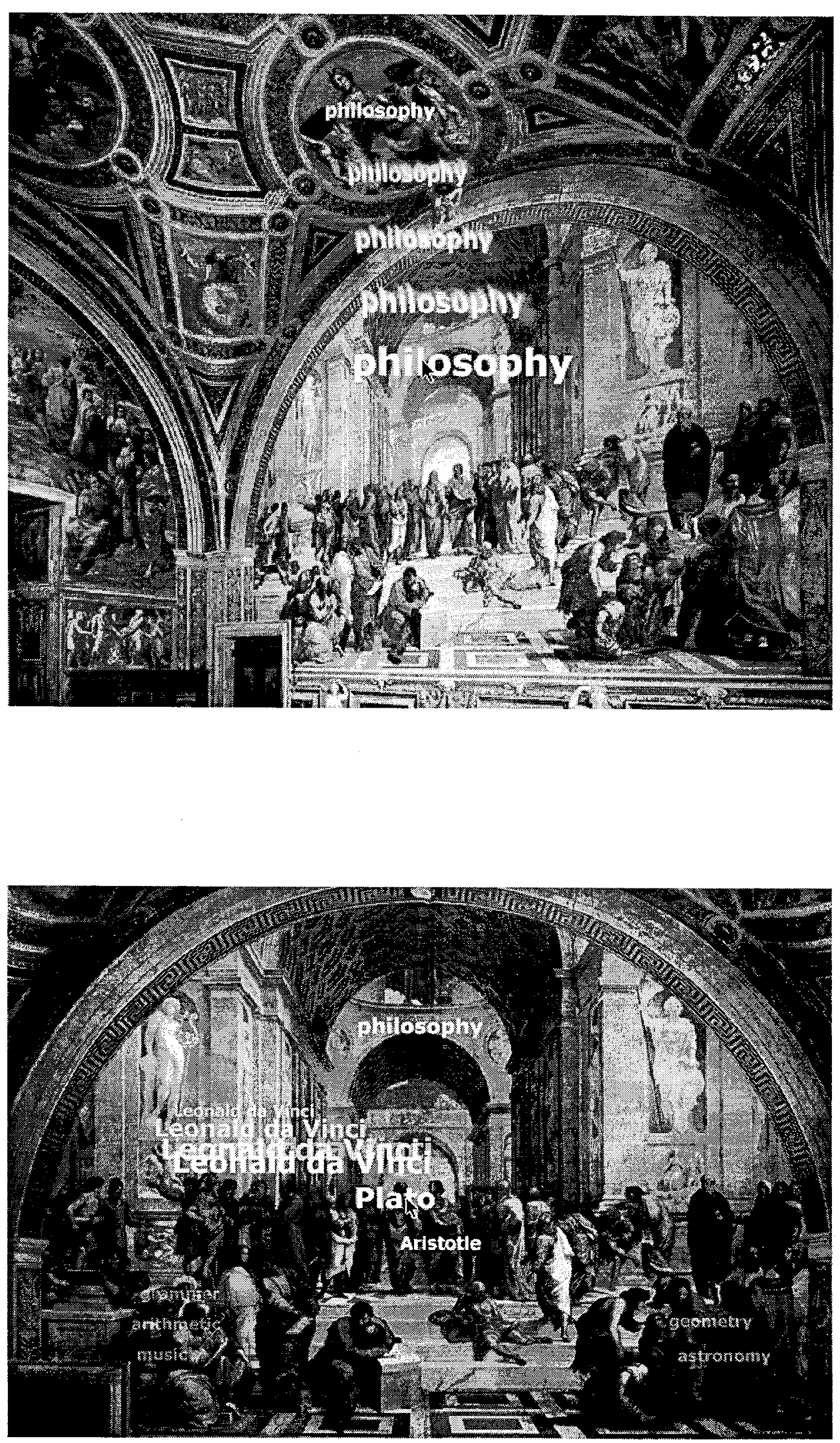
phy showing the Detailes of "School of Athens" 


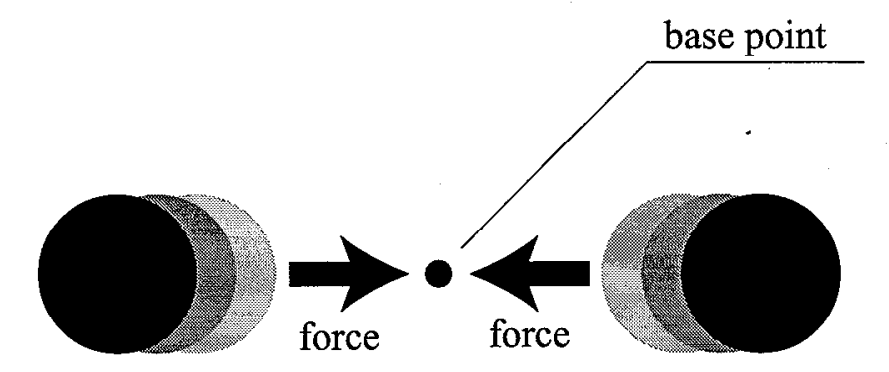

\author{
force $=$ mass $*$ acceleration \\ velocity $=$ formerly velocity + acceleration $*$ time \\ displacement $=$ formerly velocity $*$ time $+0.5 *$ acceleration $*$ time $*$ time
}

Fig.9 Script Model of Kinetic Typography

holding two books are painted on the ceiling of this room. One of the books is stood and its title is "morality." The other book is laid and its title is "nature." Philosophy is the intellectual pursuit to morality and nature. Therefore, this lady's statue is a philosophical allegory. $>$

Next, Masunari [3] explains the details of this picture. $<$ Among two men who are standing in the center on the higher floor, a man holds the book of the title "Timaeus" and is pointing at the ceiling. The other man holds the book of the title "ethics", and is pointing at the bottom a little. The former is Plato. He asked for the cause of all things at the place exceeding actuality. The latter is Aristotle. He explained to look im the face to actuality. $>$

Furthermore, the Bruno Santi [4] explains details like below.

$<$ The statue of Apollo and Minerva is the symbol of arts and sciences. That is, the front groups are people belong to "grammar", "arithmetic", and "music." The groups of front right-hand side are people belomg to "geometry" and "astronomy." And back groups are people belong to "rhetoric" and "logic."

Furthermore, the Bruno Santi adds the description. $<$ Raphael was painting several persons with the looks of people who lived in his time. Bramante is painted as Euclid. Plato will be Leonardo as pointed out probably. And finally Michelangelo is painted as Heraclitus. He sits down on stairs, and pokes an elbow on the lump of a marble, and is lost in thought gloomily. $>$

In order for an observer to understand this picture, he needs to understand the meaning of the picture. Probably, it is difficult to understand the complicated meaning of the picture simply, even if he stands in front of the real work.

\section{EXAMINATION OF DISPLAY FOR KINETIC TYPOGRAPHY}

In this Internet art museum, the picture explained above can be expressed using kinetic typography. The explanation considers the interaction method for understanding. The explanation in connection with the whole picture is shown in Fig. 7, and the kinetic typography "philosophy" shows the theme of a picture. Explanation of the details is shown in Fig. 8. The kinetic typography which shows the appearance person explains each situation.

The contents shown below explain how kinetic typography is designed in the picture.

1) The theme "philosophy" of the picture is suggested according to the book "the morality" and "nature" which the lady has. Each word of "philosophy", "morality", and "nature" is displayed as a motion linked by kinetic typography. The three words drift automatically slowly, in order to make the whole theme suggest. If one word is stimulated by a mouse, the other two words will be followed and will be moved. This motion expresses the theme of this picture visually, when each word is mutually related.

2) In explanation of the details of this picture, when bringing a mouse pointer close to Plato and Aristotle who are located in a center, appear for explanation of kinetic typography. The kinetic typography "Plato" is linked to the word "idea" with the name of the book "Timaeus." Furthermore, the kinetic typography "Aristotle" is linked to the word "systematize the world" with the name of the book "ethics."

3) In explanation of another details, when a mouse pointer close to the front group of the picture, the kinetic typography of "grammar", "arithmetic", and "music" appears. When a mouse pointer close to the right group of the picture, the kinetic typography of "geometrical" and "astronomy" appears. And when a mouse pointer close to the upper group of the picture, the kinetic typography "rhetoric" and "logic" appears.

4) When a mouse pointer close to other persons, the kinetic typography relating with each person appears.

\section{METHOD OF KINETIC TYPOGRAPHY EXPRESSION}

The element of "interaction" and "natural motion" will be important for kinetic typography. In order to design kinetic typography, authoring software is used well. This software is aimed at making the film animation like flipbook. This is the same method as making every one frame of a movie film. The design of the kinetic typography by use of authoring software is the same as that of handmade character animation. In this case, it is difficult to produce many samples of kinetic typography.

Therefore, in this research, the method of expressing kinetic typography in a program script was devised. The sample of the Internet art museum using kinetic typography was made by C-language programming.

A motion of kinetic typography is designed in an actual motion of "approach" or "expansion" by reference. The foun- 
dations of the actual motion follow a physical law. Then, for the efficient display of kinetic typography, the physical law was applied to the motion of kinetic typography. That is, the physical law of "tension", "diffusion", and "nimbleness" describe fundamental motions of kinetic typography. The equation of a physical law can cxpress kinctic typography by smooth motion rather than it is designed by hand. However, it is not efficient to program depend on each kinetic typography motions. Then, some variables are prepared, and it unites and combines with a motion of kinetic typography. As shown in Fig. 9, these motions are operated with the combination of two equations and some variables. The combination of the variable of "power", "direction", and "time" enables us to express the natural motion whose prediction is not fixed. Kinetic typography can be expressed as a script by combining two or more these motions. This format may become the standard file format of kinetic typography in the future.

\section{CONCLUSION}

This research showed a possibility that kinetic typography could be used for explanation of the exhibit works to which the interaction was applicd in the Internet art museum. And kinetic typography shows how to carry out visual presentation of the meaning which an exhibit work has. Next, there is a method of making a motion of kinetic typography generates automatically with a script. This is a possibility of appreciating the Internet art museum which used kinetic typography on the network. Furthermore, kinetic typography can visualize and present the information of a meaning which is not in sight. This is the possibility that a computer can express visually a structure of the logic which is not visible, and sensible information.

This research is funded under research grant by the Okawa Foundation.

\section{REFERENCES}

[1] One of the Japanese features has many words of onomatopoeia and mimesis compared with other foreign languages. Onomatopoeia and mimesis is effective in expressing feeling and a situation sensuously. Such language is exact material in order to consider the kinetic typography which can perform expression of a sensuous motion. Moreover, when learning Japanese, there is a possibility that kinetic typography will help hearing impairment children on study of the onomatopoeia and mimesis words.

[2]Sakae Hasegawa, Korekara no Bijutsukan, Kajima Institute Publishing Co., Itd., 1982, pp87

[3]Takashi Masunari, Viewpoints and Human KANSEI in the Appreciation of Paintings, Report of Modeling the Evaluation Structure of KANSEI 1997, University of Tsukuba, 1997, pp43-47

[4]Bruno Santi, Trancelated by Hiroshi Ishihara, RAFFAELLO, Grand Maestri Dell'arte 20, Tokyo Shoseki Co., 1995, pp28-29 